\title{
Investigación
}

\section{Criterios industriales en la transferencia de tecnología química*}

\section{Criteria in Industrial Chemistry Technology Transfer}

\section{Resumen}

Se realiza un análisis teórico de los indicadores que intervienen en la transferencia tecnológica, haciendo énfasis en aquellos de carácter industrial, logrando proponer 28 criterios industriales a partir de una primera corrida del ciclo metodológico basado principalmente en el método de investigación acción. Los criterios propuestos abarcan los procesos industriales, información cinética y termodinámica, servicios, especificaciones técnicas, control y equipos industriales. Fueron validados a través de la evaluación de las características asociadas a las métricas propuestas. Se concluye que todas las métricas son pertinentes, factibles, profundas y de adecuada escala por tener un porcentaje promedio de aceptación mayor al $75 \%$ tal como lo establece el procedimiento de evaluación.

\section{Palabras clave}

Transferencia de tecnología, criterios industriales, tecnología química, industria química.

Artículo de investigación, producto derivado del proyecto de investigación Propuesta de modelo sistémico de adopción de tecnología química, realizado en el grupo Unidad de Gestión Desarrollo Empresarial y Tecnológico de la Universidad Simón Bolívar, llevado a cabo entre el 2005 y el 2014.

** Magíster en Ingeniería de Sistemas, Ingeniero químico. Profesor Agregado a dedicación exclusiva de la Universidad Simón Bolívar, investigador en Transferencia de Tecnología Química, Gasificación de la Biomasa y Enseñanza de la Termodinámica. Estudiante del Doctorado en Ingeniería Química. Coordinador de tecnología mecánica, mantenimiento aeronáutico e ingeniería de mantenimiento en la Universidad Simón Bolívar. E-mail: martinduran@usb.ve,martinenriqueduran@gmail.com 


\section{Abstract}

A theoretical analysis of the indicators involved in technology transfer, emphasizing those industrial character, achieving 28 industrial propose criteria from a first run of methodological cycle based mainly on action research method is performed. The proposed criteria include industrial processes, kinetic and thermodynamic information, services, specifications, control and industrial equipment. Were validated by assessing the characteristics associated with the metric proposed. We conclude that all metrics are relevant, feasible, and appropriate scale deep by having an average percentage of $75 \%$ greater acceptance as established assessment procedure.

\section{Keywords}

Transfer of technology, industry standards, chemical technology, chemical industry.

\section{Introducción}

La transferencia de tecnología en la actualidad es una práctica de vital importancia en las industrias y empresas de los diferentes países del mundo. Es un proceso integral que involucra desde la selección de la tecnología hasta su adecuación en la industria, constituyéndose en una estrategia que agrega valor independientemente si la empresa, organización o país es generador o no de tecnologías.

Como estrategia, la transferencia tecnológica contribuye con el éxito de la política tecnológica en la industria (Martínez, 1998), (Antunes, Souza y Dutra, 2001) y (DuránGarcía, 2007), minimizando las pérdidas y mejorando los resultados de los diferentes procesos industriales. Sin embargo, la complejidad tecnológica en las industrias constituye un reto que afrontar en virtud del carácter multidisciplinario que la reviste.

Un claro ejemplo de organizaciones con una elevada complejidad tecnológica es la industria química, y el proceso de transferencia tecnológica dentro de esta se constituye en un aspecto vital donde se deben tomar en cuenta diversos criterios que permitan lograr con éxito la inserción adecuada de una tecnología en un contexto dado.

Específicamente cuando la industria química promueve la transferencia tecnológica en los procesos de producción, en busca del aumento de la calidad y competitividad de los productos se consiguen con presiones ecológicas y ambientales, que los conduce a la necesidad de disminuir la cantidad y mejorar la calidad de los efluentes industriales y reducir el consumo de recursos energéticos y recursos naturales (Antunes, Souza y Dutra, 2001) y (Pirela y Paredes, 2002).

Por razones de esta índole, la industria química no se escapa de la dinámica cambiante que existe en las organizaciones debido a la complejidad tecnológica antes mencionada y cada día surge la necesidad de mejorar procesos dentro de la misma, lo que hace necesario estudiar los criterios industriales y termodinámicos que repercuten en la transferencia de la tecnología química en la industria y su contexto. 
Esto conduce a formular la siguiente pregunta: ¿Por qué es prioritario establecer los criterios industriales en la transferencia de la tecnología química? El proceso de transferencia tecnológica es un proceso sistémico, donde la complejidad de las interrelaciones entre los aspectos que la involucran no son independientes unos de los otros.

Sin embargo, se observa en la práctica tecnológica de las industrias químicas que adquieren tecnologías obedeciendo a políticas aisladas que responden a un criterio o un grupo de criterios en particular: tecnología de vanguardia, tecnología necesaria a la industria, o es la más económica, entre otros, evidenciando la falta de claridad de criterios industriales que permitan definir de forma sistémica la transferencia tecnológica.

Para seleccionar una tecnología química hay que tomar en cuenta entre los criterios de selección aspectos industriales relacionados con el uso de las fuentes de energía, cantidad de materia prima, efluentes, descargas al ambiente, rangos de presiones y temperatura de operación en la planta, indicadores de tiempos de reacción química, procesos de tratamiento de productos para que no impacten en el medio ambiente, procesos de separación, plantas de tratamiento, dispositivos de almacenamiento de productos y materia prima, etc.

Estos criterios industriales impactarán sistémicamente en el proceso de transferencia de tecnología en la industria química, a pesar de la especificidad y carácter técnico de los mismos; pues contribuirán con el establecimiento de criterios que se relacionen con otros aspectos como mano de obra calificada, personal administrativo, políticas organizacionales, procesos de gestión, procesos de soporte, sistemas industriales de control, servicios técnicos, etc.

\subsection{Transferencia de la tecnología química, una realidad global}

En distintas partes del mundo se observa, que el concepto de transferencia de la tecnología, presenta diferentes connotaciones, las cuales proporcionan aportes significativos. Específicamente, se asume el concepto propuesto por (Durán-García, 2007), el cual la define como un proceso que involucra la selección, adquisición e incorporación de una tecnología, de manera que proporcione soluciones técnicas integrales a la organización en pro de sus objetivos.

En cuanto a la transferencia de la tecnología química, se tiene que el escenario químico internacional está actualmente dominado por grandes empresas, diversificadas e integradas, que conviven con empresas muy especializadas de menor tamaño. Europa, Estados Unidos y Japón, concentran 85 \% del mercado y reciben los principales flujos de inversiones directas de la industria (Antunes, Souza y Dutra, 2001).

Aunado a esto, en la transferencia de tecnología, los países se han encontrado con serios inconvenientes entre los cuales, se mencionan algunos:

- Cláusulas restrictivas en los convenios de transferencia, como los sistemas de patentes.

- Adquisición de tecnologías obsoletas y costosas.

- Adquisición de tecnologías que no se adaptan al contexto de la organización receptora. 
En la industria química de Brasil y Venezuela, se observa el manejo de la patente como estrategia tecnológica, esto se muestra en un estudio realizado donde una de las conclusiones más importantes de este trabajo es que las empresas estatales de Venezuela y Brasil son las mayores detentoras de patentes, donde INTEVEP concentra el $77 \%$ de las patentes en las industrias químicas venezolanas y PETROBRAS tiene el $30 \%$ (Antunes, Souza y Dutra, 2001).Esto constituye un criterio de importancia para las organizaciones que deseen insertarse en el proceso de transferencia de la tecnología química, pues se necesita una adecuada capacidad de negociación con organizaciones de estados que poseen la mayoría de patentes y/o licencias.

En opinión de (Martínez, 1998), (Durán-García, 2007), (Álvarez, Rincón, Pérez y Hernández, 2008) y (Turton, Bailie, Whiting, Shaeiwitz y Bhattacharyya, 2012), los inconvenientes más comunes en la transferencia de tecnología, son los siguientes:

- Desconocimiento de los diferentes tipos de tecnologías al momento de seleccionar, negociar y adquirir una tecnología.

- Ausencia de un estudio previo que permita por lo menos la adecuada selección y adquisición de una tecnología.

- Obligación por parte de la organización adquirente, de mantener en secreto el conocimiento que se transfiere, más allá de la vigencia del contrato de comercialización de tecnología.

- Legislación aplicable y tribunales competentes a la organización que adquiere la tecnología, en virtud de las posibles diferencias legales que se produzcan. Los aspectos legales deben ser resueltos según las leyes del país de la organización que cede tecnología.

Este esquema de transferencia lejos de impulsar el desarrollo integral entre ambas partes, propicia una dependencia perjudicial y condiciones antieconómicas, principalmente para la parte que adquiere tecnología. El escaso balance positivo para la parte adquirente se traduce, en términos generales, en un proceso de transferencia con unos escasos matices de asesoría técnica y una negociación más o menos favorable a pesar de las condiciones restrictivas.

En la industria de tratamiento de agua, (Galvis y Vargas, 1998) mencionan que la selección de la tecnología debe estar enmarcada en el concepto de sostenibilidad, como respuesta a las deficiencias en las tecnologías existentes en los sistemas de tratamiento de agua. Entendiéndose por un sistema sostenible aquel que suministra un servicio con criterio de calidad y eficiencia económica y ambiental, el cual puede ser financiado o cofinanciado por sus usuarios (Galvis y Vargas, 1998). Se evidencia de tal forma que el adecuado proceso de selección y adopción de la tecnología química, involucra las dimensiones de ambiente, tecnología y comunidad, las cuales se interrelacionan en el contexto político, socioeconómico, cultural e institucional. Todo esto hace a la adopción de la tecnología química un complejo proceso de toma de decisión.

Según (Pirela y Paredes, 2002) y (Turton, Bailie, Whiting, Shaeiwitz y Bhattacharyya, 2012), las tecnologías químicas cambian con el tiempo, y varían de acuerdo con el campo 
de conocimiento o sector industrial, y las diferencias entre un país y otro, con relación a las tecnologías químicas, dependen de la historia de la cultura de cada país y del momento al que se incorporó al proceso de industrialización. Esta idea la complementan (Antunes, Souza y Dutra, 2001) los cuales explican que las transformaciones ocurridas en los procesos y productos químicos en los últimos años, son de tal magnitud, que pueden hacer desaparecer grupos enteros de industrias químicas, por venir a ser considerados obsoletos y de costos no competitivos. Hay que manejar este tipo de situaciones a través de un serio análisis que conlleve a la adecuada toma de decisión en el proceso de transferencia tecnológica que proponga criterios claros entre los cuales se encuentran los criterios industriales.

\section{Metodología}

Se proponen entonces las actividades a ser llevadas a cabo, con el fin de alcanzar el objetivo planteado, a través de un marco metodológico (Pérez, Rojas, Mendoza y Grimán, 2004), que se basa principalmente en el método de Investigación Acción y la metodología DESMET. Se abordan los primeros siete pasos del ciclo metodológico que originan como producto los criterios industriales en la transferencia de la tecnología química y sus respectivas métricas.

Estos criterios están enmarcados dentro del Modelo sistémico de adopción de tecnología química propuesto (Durán-García, 2007), el cual ubica ámbitos sociales, técnicos, ambientales, industriales, culturales, organizacionales, científicos que intervienen en el proceso de transferencia tecnológica. Este ciclo propuesto puede repetirse $n$ veces, pero para efectos de esta investigación se considera una sola iteración en virtud de que no se están tomando en cuenta todas las actividades de la primera iteración, ver tabla I.

Tabla I. Fases de la Metodología investigación acción propuesta. Fuente: Adaptado de (Pérez, Rojas, Mendoza y Griman, 2004) y (Durán-García, 2007).

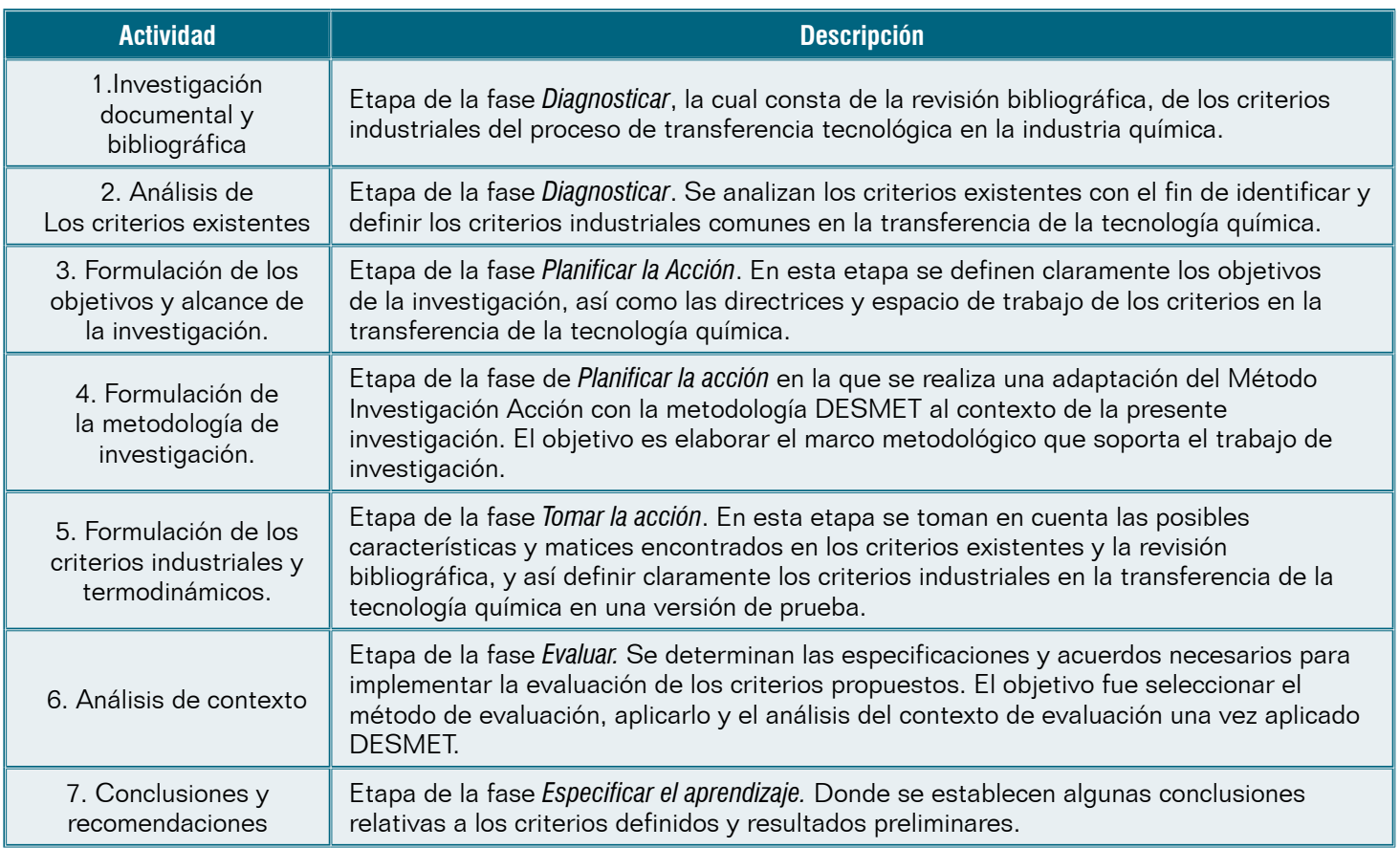




\section{Análisis y discusión de resultados}

A partir de la selección y análisis de los criterios usados comúnmente en la práctica de ingeniería (conocimiento adquirido con la experiencia por expertos en el manejo de la adopción tecnológica, prácticas básicas, etc.) que contribuyen con una adecuada transferencia, los modelos teóricos, y los aportes de la literatura (Antunes, Souza y Dutra, 2001), (Rivera, 2002), (Álvarez, Rincón y Pérez, 2005), (Mendoza, Pérez y Griman, 2005), (Rincón, Pérez y Álvarez, 2005), (Rincón, 2006), (Durán-García, 2007), (Álvarez, Rincón, Pérez y Hernández, 2008), (Durán-García, Pérez, Rincón y Mendoza, 2011), (Turton, Bailie, Whiting, Shaeiwitz y Bhattacharyya, 2012) y (Durán-García, 2014), se han conceptualizado 28 criterios de carácter industrial dentro un Modelo sistémico de adopción de la tecnología por la industria química propuesto por (Durán-García, Pérez, Rincón y Mendoza, 2011), los cuales se presentan en la tabla II. Estos criterios se encuentran dentro de la "dimensión ingeniería» que interacciona sistémicamente con las dimensiones organizacional, gestión, cliente-proveedor y soporte propuesta por (Durán-García, Pérez, Rincón y Mendoza, 2011).

Tabla II. Criterios industriales y termodinámicos en la transferencia de la tecnología química. Fuente: Adaptado de (Durán-García, Pérez, Rincón y Mendoza, 2011).

\begin{tabular}{|c|c|c|}
\hline \multirow{5}{*}{$\begin{array}{l}\text { Información cinética y termodinámica } \\
\text { (ICT) }\end{array}$} & \multicolumn{2}{|c|}{ Criterios industriales y termodinámicos } \\
\hline & ICT 01 & Información de la reacción química \\
\hline & ICT 02 & Desempeño de la reacción química \\
\hline & ICT 03 & Procesos termodinámicos \\
\hline & ICT 04 & Condiciones de operación de la reacción química \\
\hline \multirow{6}{*}{ Procesos industriales (PRI) } & PRI 01 & Diagrama de los procesos productivos y conexos \\
\hline & PRI 02 & Compuestos no deseados \\
\hline & PRI 03 & Sustentable energéticamente \\
\hline & PRI 04 & Productos y subproductos \\
\hline & PRI 05 & Condiciones de operación de equipos y accesorios \\
\hline & PRI 06 & Impacto en los procesos \\
\hline \multirow{6}{*}{ Equipos (EQU) } & EQU 01 & Equipos y accesorios \\
\hline & EQU 02 & Manuales de uso \\
\hline & EQU 03 & $\begin{array}{l}\text { Características propias de los equipos y } \\
\text { accesorios }\end{array}$ \\
\hline & EQU 04 & Plantas de tratamiento \\
\hline & EQU 05 & Efluentes \\
\hline & EQU 06 & Contaminación sónica \\
\hline \multirow{4}{*}{$\begin{array}{l}\text { Especificaciones } \\
\text { (ESP) }\end{array}$} & ESP 01 & Especificación de materia prima \\
\hline & ESP 02 & Especificación de insumos \\
\hline & ESP 03 & Especificación del producto \\
\hline & ESP 04 & Desechos sólidos y residuos contaminantes \\
\hline \multirow{4}{*}{ Servicio (SER) } & SER 01 & Energía \\
\hline & SER 02 & Disponibilidad de corrientes de alimentación \\
\hline & SER 03 & Almacenaje \\
\hline & SER 04 & Emisiones gaseosas \\
\hline
\end{tabular}




\begin{tabular}{|l|l|l|}
\hline \multirow{2}{*}{ Control (CON) } & \multicolumn{2}{|c|}{ Criterios industriales y termodinámicos } \\
\cline { 2 - 3 } & CON 01 & Automatización \\
\cline { 2 - 3 } & CON 02 & Dispositivos de medición \\
\cline { 2 - 3 } & CON 03 & Arranque y parada \\
\cline { 2 - 3 } & CON 04 & Sistemas automatizados \\
\hline
\end{tabular}

Característica: Información cinética y termodinámica (ICT). Se refiere a las especificaciones de cinética, termodinámica y de equilibrio de todas las especies involucradas en los procesos industriales.

ICT 01. Información de la reacción química. Es la información necesaria que permita el diseño de una adecuada reacción química en el ámbito industrial en función de las bases de diseño: condiciones de operación de la reacción química en contextos similares (temperatura, presión, fracción molar y másica), cinética de la reacción y configuración del reactor.

ICT 02. Desempeño de la reacción química. Es el rendimiento de la reacción química en el ámbito industrial en función de las bases de diseño.

ICT 03. Procesos termodinámicos. Disponibilidad de procesos termodinámicos que se pueden realizar a través de diferentes operaciones químicas en la planta: equilibrio líquido -vapor, equilibrio líquido- líquido, sistemas de no equilibrio, monitoreo de propiedades termodinámicas en procesos de cambios de fase, rendimiento energético, eficiencia de ciclos termodinámicos, etc.

ICT 04. Condiciones de operación de la reacción química. Se refiere a las condiciones de operación y desempeño de la reacción química: temperatura, presión, fracción molar y másica.

Característica: Procesos industriales (PRI). Se refiere a los aspectos importantes de los procesos industriales tales como: sustentabilidad energética, documentación de los procesos, productos y compuestos no deseados, etc.

PRI 01. Diagramas de los procesos productivos y conexos. Se refiere a la documentación de la planta química que representa los procesos industriales, balance de masa y energía, espacio físico a utilizar, etc.

PRI 02. Compuestos no deseados. Se refiere al manejo y disposición de los tipos de compuestos no deseados que se generan en la planta tales como: compuestos tóxicos, compuestos corrosivos, etc.

PRI 03. Sustentable energéticamente. Si los procesos industriales son sostenibles en el contexto real de la planta química, a partir de la disponibilidad de las diferentes fuentes de energía.

PRI 04. Productos y subproductos. Se refiere a los productos y subproductos generados en los diferentes procesos industriales. 
PRI 05. Condiciones de operación de equipos y accesorios. Se refiere a las especificaciones de presión y temperatura de operación de los equipos y accesorios donde se realizan las operaciones unitarias.

PRI 06. Impacto en los procesos. Referido a los efectos que se producen en los procesos asociados (destilación, filtración, secado, evaporación, etc.): mejoras, cambios parciales en los mismos, reestructuraciones, eliminación de subprocesos, etc.

Los criterios presentados en las características ICT y PRI representan las relaciones más adecuadas entre todas las variables técnicas de ingeniería que intervienen en la transferencia tecnológica y el impacto sobre variables organizacionales, culturales, sociales, ambientales de la industria química que desea transferir tecnológicamente.

Estos pueden ser refinados con sus métricas respectivas de ser necesario en una fase posterior de la investigación si el método de evaluación lo especifica. La refinación de los criterios dependerán de los porcentajes promedio de aceptación de las características de pertinencia, factibilidad, escala y profundidad adecuada para cada una de las métricas propuestas (Álvarez, Rincón y Pérez, 2005), (Rincón, Pérez y Álvarez, 2005), (Durán-García, 2007), (Álvarez, Rincón, Pérez y Hernández, 2008) y (Durán-García, 2014).

Característica: Equipos (EQU). Se refiere a las especificaciones de diseño físico, documentación, características intrínsecas, descargas y tipos de equipos donde se realizan las operaciones unitarias: torres de destilación, intercambiadores de calor, tanques, torres de adsorción, bombas, evaporadores, turbinas, reactores químicos, compresores, etc.

EQU 01. Equipos y accesorios. Se refiere a las especificaciones de diseño físico de los equipos y accesorios de los equipos: tanques, válvulas, torres de destilación, tuberías, bombas, conexiones de equipos, evaporadores, turbinas, etc.

EQU 02. Manuales de uso. Se refiere a la documentación que describe las características de cada equipo y accesorios a través de una nomenclatura estándar.

EQU 03.Características propias de los equipos y accesorios. Se refiere a las condiciones intrínsecas de cada equipo y accesorio, como los rangos de potencia de las bombas y compresores, orientación de los reactores, tuberías y torres de separación (vertical y horizontal), etc.

EQU 04. Plantas de tratamiento. Disponibilidad de plantas de tratamiento de agua y gases, como consecuencia de la instalación de la nueva planta o tecnología insertada en la misma

EQU 05. Efluentes. Se refiere al manejo y disposición de los efluentes que se generan en la planta.

EQU 06. Contaminación sónica. Manejo del nivel de ruido generado por la planta que afecta al personal que labora en la misma y al entorno circundante. 
Característica: Especificaciones (ESP). Se refiere a las especificaciones de insumos, materia prima, desechos y productos de acuerdo a las bases de diseño.

ESP 01. Especificación de materia prima. Se refiere al cumplimiento de las especificaciones de la materia prima propuesta en las bases de diseño.

ESP 02. Especificación de insumos. Se refiere al cumplimiento de las especificaciones de los insumos, necesarios en la planta química, propuestos en las bases de diseño.

ESP 03.Especificación del producto. Se refiere al cumplimiento de las especificaciones de los productos que se generan en la planta química, propuestos en las bases de diseño.

ESP 04. Desechos sólidos y residuos contaminantes. Se refiere a las especificaciones y manejo de los contaminantes que se generan de los procesos asociados a la planta química que perjudican el medio ambiente.

Característica: Servicios (SER). Se refiere al cumplimiento de las especificaciones de los servicios, necesarios en la planta química, propuestos en las bases de diseño.

SER 01. Energía. Es la disponibilidad real de fuentes de energía: electricidad de calentamiento (electricheat, el), gas natural (natural gas, ng), gas combustible (fuel gas, $f g$ ), combustible (fuel oil, fo), agua contra fuego (firewater, fw), etc., necesarias en las operaciones de la planta según las bases de diseño.

SER 02. Disponibilidad de corrientes de alimentación. Es la disponibilidad real de las secuencias de transferencia energética que circulan y abastecen a las unidades y equipos de la planta tales como: vapor de baja presión (lowpressuresteam, Ips), vapor de alta presión (highpressuresteam, hps), agua refrigerante (refrigeratedwater, rw), etc., necesarias en las operaciones de la planta según las bases de diseño.

SER 03. Almacenaje. Es la disponibilidad real y el lugar de instalación de almacenaje del inventario de materia prima y productos que se generan en la planta química.

SER 04. Emisiones Gaseosas. Manejo e inventario de las emisiones gaseosas en función de su capacidad productiva y las regulaciones en beneficio del ambiente.

Característica: Control (CON). Disponibilidad de sistemas de control que garanticen el adecuado funcionamiento de los procesos y las unidades de operación, a partir de las especificaciones de las bases de diseño.

CON 01. Automatización. Indica el grado de automatización de las operaciones que realiza la tecnología química.

CON 02. Dispositivos de medición. Se refiere a la disponibilidad de dispositivos que permitan tomar las medidas de variables importantes dentro de los procesos industriales que se realizan en la planta. 
CON 03. Arranque y parada. Se refiere a los sistemas de control asociados al arranque y parada de la planta. Impacto de la tecnología adquirida en los equipos y las tecnologías asociadas a medida que la planta alcanza el estado estacionario a partir del arranque, y cuando se somete a la parada. Comportamiento de la planta ante procesos inesperados debido a cambios imprevistos en las condiciones de operación.

CON 04. Sistemas automatizados. Asociado a la disponibilidad de sistemas de computación que permitan reflejar y controlar los procesos industriales que se realizan en las diferentes unidades y equipos de operación.

Los criterios presentados en las características EQU, ESP, SER y CON representan las relaciones más adecuadas entre todas las variables industriales y su impacto en los otros ámbitos que intervienen en la transferencia tecnológica. Son 28 los criterios industriales establecidos que de igual forma serán refinados de acuerdo a la validación de sus métricas asociadas (Pérez, Rojas, Mendoza y Griman, 2004), (Álvarez, Rincón y Pérez, 2005), (Rincón, Pérez y Álvarez, 2005), (Durán-García, 2007), (Álvarez, Rincón, Pérez y Hernández, 2008) y (Durán-García, 2014).

A su vez se presenta un ejemplo de la formulación de las métricas con sus preguntas correspondientes para la evaluación de los criterios de Información cinética y Termodinámica y Procesos industriales de manera que el criterio sea adecuado y pertinente para el proceso de transferencia de tecnología química en la tabla III y IV respectivamente.

Tabla III. Métricas de algunos de los criterios de Información cinética y Termodinámica. Fuente: Adaptado de (Durán-García, Pérez, Rincón y Mendoza, 2011).

\begin{tabular}{|c|c|c|c|}
\hline Criterio & Métrica & Pregunta & Formulación \\
\hline \multicolumn{4}{|c|}{ ICT. Información cinética y termodinámica } \\
\hline \multirow{4}{*}{$\begin{array}{l}\text { Información de la } \\
\text { reacción química } \\
\text { ICT } 01\end{array}$} & $\begin{array}{l}\text { Condiciones de operación de la } \\
\text { reacción química. }\end{array}$ & $\begin{array}{c}\text { ¿Se documentaron las especificaciones de } \\
\text { las condiciones de operación de la reacción } \\
\text { química? }\end{array}$ & $\begin{array}{l}0=\text { No } \\
1=\text { Sí }\end{array}$ \\
\hline & Cinética de la reacción. & $\begin{array}{l}\text { ¿Se documentaron las especificaciones de la } \\
\text { cinética de reacción? }\end{array}$ & $\begin{array}{l}0=\text { No } \\
1=\text { Sí }\end{array}$ \\
\hline & Fracción molar y másica. & $\begin{array}{c}\text { ¿Se justificó por escrito la necesidad de conocer } \\
\text { la fracción molar y másica de las especies } \\
\text { químicas? }\end{array}$ & $\begin{array}{l}0=\text { No } \\
1=\text { Sí }\end{array}$ \\
\hline & Configuración del reactor. & $\begin{array}{l}\text { ¿Se documentó la especificación de la } \\
\text { configuración del reactor? }\end{array}$ & $\begin{array}{l}0=\text { No } \\
1=\text { Sí }\end{array}$ \\
\hline \multirow{2}{*}{$\begin{array}{l}\text { Desempeño de la } \\
\text { reacción química } \\
\text { ICT } 02\end{array}$} & $\begin{array}{l}\text { Rendimiento de la reacción } \\
\text { química. }\end{array}$ & $\begin{array}{l}\text { ¿Se documentó la evaluación del rendimiento de } \\
\text { la reacción química? }\end{array}$ & $\begin{array}{l}0=\text { No } \\
1=\text { Sí }\end{array}$ \\
\hline & $\begin{array}{l}\text { Pureza de los productos } \\
\text { obtenidos. }\end{array}$ & $\begin{array}{l}\text { ¿Se documentó la evaluación de la pureza de los } \\
\text { productos obtenidos? }\end{array}$ & $\begin{array}{l}0=\text { No } \\
1=\text { Sí }\end{array}$ \\
\hline \multirow{2}{*}{$\begin{array}{l}\text { Procesos } \\
\text { Termodinámicos } \\
\text { ICT } 03\end{array}$} & $\begin{array}{l}\text { Disponibilidad de procesos } \\
\text { termodinámicos. }\end{array}$ & $\begin{array}{l}\text { ¿Se documentaron los procesos } \\
\text { termodinámicos que se pueden ejecutar con la } \\
\text { nueva tecnología? }\end{array}$ & $\begin{array}{l}0=\text { No } \\
1=\text { Sí }\end{array}$ \\
\hline & $\begin{array}{l}\text { Eficiencia de los procesos } \\
\text { termodinámicos. }\end{array}$ & $\begin{array}{l}\text { ¿Se documentó el análisis de la eficiencia de los } \\
\text { procesos termodinámicos? }\end{array}$ & $\begin{array}{l}0=\text { No } \\
1=\text { Sí }\end{array}$ \\
\hline
\end{tabular}


Tabla IV. Métricas de los criterios de procesos industriales. Fuente: Adaptado de (Durán-García, Pérez,

Rincón y Mendoza, 2011).

\begin{tabular}{|c|c|c|c|}
\hline Criterio & Métrica & Pregunta & Formulación \\
\hline \multicolumn{4}{|c|}{ Procesos industriales PRI } \\
\hline \multirow{2}{*}{$\begin{array}{l}\text { Diagrama de los } \\
\text { procesos productivos y } \\
\text { conexos PRI } 01\end{array}$} & $\begin{array}{l}\text { Diagrama de procesos } \\
\text { industriales. }\end{array}$ & $\begin{array}{l}\text { ¿Se elaboraron los diagramas de procesos } \\
\text { industriales? }\end{array}$ & $\begin{array}{l}0=\text { No } \\
1=\text { Sí }\end{array}$ \\
\hline & $\begin{array}{l}\text { Diagrama de procesos } \\
\text { conexos. }\end{array}$ & ¿Se elaboraron los diagramas de procesos conexos? & $\begin{array}{l}0=\text { No } \\
1=\text { Sí }\end{array}$ \\
\hline \multirow{2}{*}{$\begin{array}{l}\text { Compuestos no } \\
\text { deseados } \\
\text { PRI } 02\end{array}$} & $\begin{array}{l}\text { Información de los } \\
\text { compuestos no deseados. }\end{array}$ & $\begin{array}{l}\text { ¿Se documentaron las especificaciones de los } \\
\text { compuestos no deseados que se generan? }\end{array}$ & $\begin{array}{l}0=\text { No } \\
1=\text { Sí }\end{array}$ \\
\hline & $\begin{array}{l}\text { Aprovechamiento de los } \\
\text { compuestos no deseados. }\end{array}$ & $\begin{array}{l}\text { ¿Se documentó el aprovechamiento de los } \\
\text { compuestos no deseados? }\end{array}$ & $\begin{array}{l}0=\text { No } \\
1=\text { Sí }\end{array}$ \\
\hline \multirow{2}{*}{$\begin{array}{l}\text { Sustentable } \\
\text { energéticamente } \\
\text { PRI } 03\end{array}$} & Demanda energética. & $\begin{array}{c}\text { ¿Se documenta el análisis de las fuentes de energía } \\
\text { disponibles que lograron satisfacer la demanda de } \\
\text { energía requerida? }\end{array}$ & $\begin{array}{l}0=\text { No } \\
1=\text { Sí }\end{array}$ \\
\hline & Energía disponible. & $\begin{array}{l}\text { ¿Se documentó la evaluación/estimación de la energía } \\
\text { que demandaban los procesos industriales? }\end{array}$ & $\begin{array}{l}0=\text { No } \\
1=\text { Sí }\end{array}$ \\
\hline \multirow{2}{*}{$\begin{array}{l}\text { Productos y } \\
\text { subproductos } \\
\text { PRI } 04\end{array}$} & \multirow{2}{*}{$\begin{array}{l}\text { Características de los } \\
\text { productos y subproductos. }\end{array}$} & $\begin{array}{l}\text { ¿Se documentaron las características de los } \\
\text { productos y subproductos esperados de acuerdo a las } \\
\text { bases de diseño? }\end{array}$ & $\begin{array}{l}1=\text { Sí } \\
0=\text { No }\end{array}$ \\
\hline & & $\begin{array}{c}\text { ¿Se documentaron las características de los } \\
\text { productos y subproductos generados por la nueva } \\
\text { planta química? }\end{array}$ & $\begin{array}{l}1=\text { Sí } \\
0=\text { No }\end{array}$ \\
\hline \multirow{2}{*}{$\begin{array}{l}\text { Condiciones de } \\
\text { operación de equipos y } \\
\text { accesorios } \\
\text { PRI } 05\end{array}$} & Presión & $\begin{array}{l}\text { ¿La especificación sobre el rango de presión } \\
\text { de operación de los equipos y accesorios fue } \\
\text { especificada? }\end{array}$ & $\begin{array}{l}0=\text { No } \\
1=\text { Sí }\end{array}$ \\
\hline & Temperatura & $\begin{array}{l}\text { ¿La especificación sobre el rango de temperatura } \\
\text { de operación de los equipos y accesorios fue } \\
\text { especificada? }\end{array}$ & $\begin{array}{l}0=\text { No } \\
1=\text { Sí }\end{array}$ \\
\hline \multirow{3}{*}{$\begin{array}{l}\text { Impacto en los } \\
\text { procesos } \\
\text { PRI } 06\end{array}$} & Mejoras en los procesos. & $\begin{array}{l}\text { ¿Se documentó la evaluación de las mejoras de los } \\
\text { procesos industriales? }\end{array}$ & $\begin{array}{l}0=\text { No } \\
1=\text { Sí }\end{array}$ \\
\hline & $\begin{array}{l}\text { Cambios y } \\
\text { reestructuraciones } \\
\text { parciales en los procesos. }\end{array}$ & $\begin{array}{c}\text { ¿Se documentó la evaluación de la pertinencia en } \\
\text { los cambios y reestructuraciones parciales de los } \\
\text { procesos? }\end{array}$ & $\begin{array}{l}0=\text { No } \\
1=\text { Sí }\end{array}$ \\
\hline & Cambios de subprocesos. & $\begin{array}{l}\text { ¿Se documentó la evaluación de los posibles cambios } \\
\text { de los subprocesos? }\end{array}$ & $\begin{array}{l}0=\text { No } \\
1=\text { Sí }\end{array}$ \\
\hline
\end{tabular}

\subsection{Procedimiento de evaluación de los criterios industriales en la transferencia de la tecnología química.}

Estos criterios son propuestos en una primera versión dentro del ciclo metodológico, por lo que una vez que sean evaluados a través de sus métricas de acuerdo al método de evaluación de la metodología DESMET se realizará la refinación de los mismos de manera que se adapten lo mejor posible al proceso sistémico de transferencia de la tecnología química (Pérez, Rojas, Mendoza y Griman, 2004) y (Durán-García, Pérez, Rincón y Mendoza, 2011).

DESMET es una metodología que permite identificar claramente el contexto existente en la evaluación de los criterios industriales, así como las limitaciones y puntos fuertes de los mismos, a través de condiciones favorables presentes y no presentes en cada uno 
de los métodos de evaluación. Esta metodología se compone de nueve métodos entre los cuales están las encuestas cuantitativas, los análisis de características por proyección, por caso de estudio, por experimento y encuestas, entre otros.

De estos se selecciona para la evaluación de los criterios actuales, el método de evaluación denominado "Análisis de características por encuestas», por contener la mayor cantidad de condiciones favorables presentes en el mismo. Este método es útil cuando es difícil cuantificar los beneficios y además cuando se conoce la población de usuarios que utilizarán los criterios, permitiendo seleccionar apropiadamente los expertos evaluadores, que hayan hecho uso de estos criterios en proyectos previos.

Una vez seleccionado el método y los evaluadores que participarán, se deben entregar (en físico o en digital) los cuestionarios a cada uno de los expertos para que evalúen los criterios en función de las métricas asociadas a cada uno (cuatro evaluadores de acuerdo al método de evaluación arrojado por DESMET). Para seleccionar los criterios definitivos, el nivel promedio de aceptación de cada grupo de métricas asociadas deberá ser del setenta y cinco por ciento (75\%), donde cada respuesta positiva dada por los expertos asumirá el valor de uno (1).

Para cuantificar si un grupo de métricas asociadas a un criterio son aceptadas o no, se deben considerar las cuatro respuestas para cada pregunta a evaluar, en función del nivel de pertinencia, factibilidad, profundidad y escala para las métricas, como se muestra en la tabla V. Si una métrica no supera el nivel mínimo promedio de aceptación del 75 \% será desechada al momento de validar los criterios industriales.

Tabla V. Características específicas para la Evaluación de cada métrica. Fuente: Adaptado de (DuránGarcía, Pérez, Rincón y Mendoza, 2011).

\begin{tabular}{|c|l|l||}
\hline Característica general & \multicolumn{1}{|c|}{ Descripción } & \multicolumn{1}{|c|}{ Escala } \\
\hline Pertinencia de la métrica & $\begin{array}{l}\text { Se refiere a si la métrica es adecuada para } \\
\text { medir la existencia o no de la característica } \\
\text { donde se encuentra. }\end{array}$ & $\begin{array}{l}\text { 1: significa que la métrica es pertinente. } \\
\text { 0: significa que la métrica no es pertinente. }\end{array}$ \\
\hline \hline Factibilidad de la métrica. & $\begin{array}{l}\text { Se refiere a si es factible medir la } \\
\text { característica propuesta en la métrica dentro } \\
\text { del contexto de evaluación. }\end{array}$ & $\begin{array}{l}\text { 1: significa que la métrica es factible. } \\
\text { 0: significa que la métrica no es factible. }\end{array}$ \\
\hline \hline Nivel de profundidad & $\begin{array}{l}\text { Se refiere a si la métrica a verificar tiene el } \\
\text { nivel de profundidad adecuado para que el } \\
\text { resultado sea relevante. }\end{array}$ & $\begin{array}{l}\text { 1: significa que la métrica tiene el nivel de } \\
\text { profundidad adecuado. } \\
\text { 0: significa que se requiere una mayor profundidad } \\
\text { en la métrica. }\end{array}$ \\
\hline \hline Escala de la métrica & $\begin{array}{l}\text { Se refiere a si la escala propuesta es } \\
\text { adecuada para medir la métrica. }\end{array}$ & $\begin{array}{l}\text { 1: significa que la escala es adecuada } \\
\text { 0: significa que la escala no es adecuada. }\end{array}$ \\
\hline
\end{tabular}

En tal sentido, las métricas asociadas a este grupo de criterios industriales son en total cuarenta y nueve (49) que permiten validarlos a partir de las características de pertinencia, factibilidad, profundidad y escala de cada métrica. Se presenta en la tabla VI los resultados de la evaluación de las métricas a partir de sus características específicas que validan los criterios industriales que se deben tener en cuenta en la transferencia de la tecnología química. 
Tabla VI. Porcentaje promedio de Evaluación de cada métrica. Fuente: Elaboración propia.

\begin{tabular}{|c|c|c|c|c|}
\hline \multirow{2}{*}{ Grupo de Criterios } & \multicolumn{4}{|c|}{ Características Específicas para la Evaluación de Cada Métrica } \\
\hline & $\begin{array}{l}\text { Pertinencia }(\% \\
\text { promedio) }\end{array}$ & $\begin{array}{l}\text { Factibilidad } \\
\text { (\% promedio) }\end{array}$ & $\begin{array}{l}\text { Profundidad (\% } \\
\text { promedio) }\end{array}$ & $\begin{array}{l}\text { Escala } \\
\text { (\% promedio) }\end{array}$ \\
\hline $\begin{array}{l}\text { Información cinética y termodinámica (ICT, } 8 \\
\text { métricas) }\end{array}$ & 100 & 100 & 80 & 86 \\
\hline Procesos industriales (PRI, 12 métricas) & 98 & 96 & 91 & 91 \\
\hline Especificaciones (ESP, 6 métricas) & 96 & 89 & 85 & 85 \\
\hline Equipos (EQU, 10 métricas) & 96 & 93 & 89 & 89 \\
\hline Servicios (SER, 7 métricas) & 96 & 96 & 93 & 89 \\
\hline Control (CON, 6 métricas) & 100 & 96 & 93 & 89 \\
\hline Promedio total (49 métricas) & 98 & 95 & 89 & 88 \\
\hline
\end{tabular}

De acuerdo a la tabla $\mathrm{VI}$, las 49 métricas asociadas a los 28 criterios industriales tienen un porcentaje de aceptación promedio por encima del $75 \%$, el cual es el nivel mínimo para que una métrica sea pertinente, factible, profunda y de adecuada escala. Esto indica que cada una de las métricas formuladas valida a sus respectivos criterios propuestos.

La mayoría de los casos, donde a lo sumo uno de los cuatros evaluadores calificó con "0» a una o varias métricas correspondientes a un grupo de métricas que valida un criterio en particular, fue en las características de profundidad y escala, por considerar que debiera tener una escala más amplia y no del tipo binaria, y además que el enunciado de la misma requiere mayor nivel de detalle garantizando su profundidad.

Sin embargo, es importante recordar que esta investigación se realiza bajo una metodología que cuantifica los resultados como aceptados o no aceptados bajo un enfoque de calidad sistémica (Pérez, Rojas, Mendoza y Griman, 2004) y (Durán-García, Pérez, Rincón y Mendoza, 2011), por lo tanto una escala que varíe progresivamente no tiene cabida dentro de la escala propuesta en esta investigación.

En cuanto a la profundidad, es razonable tener valoraciones de este tipo por alguno de los expertos, entendiendo que estos son criterios propuestos en una primera versión del ciclo de investigación, lo que obedece a una conceptualización que posiblemente escape a la mejor forma de definirlo.

No obstante, las métricas correspondientes no se eliminan pues a pesar de esa valoración nula, el promedio de aceptación del grupo de métricas asociadas a determinados criterios para las características de evaluación de profundidad y escala es mayor al mínimo de aceptación. 


\section{Conclusiones}

Basándose en los resultados obtenidos y el análisis de los mismos, es posible indicar algunas conclusiones:

La formulación de los criterios industriales y termodinámicos en la transferencia de la tecnología química se fundamentó en las más recientes investigaciones que se han realizado en el ámbito académico (Antunes, Souza y Dutra, 2001), (Rivera, 2002), (Álvarez, Rincón y Pérez, 2005), (Mendoza, Pérez y Griman, 2005), (Rincón, Pérez y Álvarez, 2005), (Rincón, 2006), (Durán-García, 2007), (Álvarez, Rincón, Pérez y Hernández, 2008), (Durán-García, Pérez, Rincón y Mendoza, 2011), (Turton, Bailie, Whiting, Shaeiwitz y Bhattacharyya, 2012) y (Durán-García, 2014) generándose como producto 28 criterios a ser evaluados con sus respectivas métricas.

La aplicación de la metodología DESMET con el método investigación acción permitió la evaluación de los 28 criterios propuestos arrojando la validación de los mismos a través de un porcentaje promedio de aceptación del $98 \%$ pertinencia, $95 \%$ factibilidad, $89 \%$ de profundidad y $88 \%$ de adecuación de escala de cada grupo de métricas asociadas a los criterios propuestos.

Esta investigación es un punto de inicio para que se propongan un mayor número de criterios que permitan evaluar adecuadamente y con mayor completitud un proceso de transferencia de tecnología química.

\section{Referencias}

Álvarez, M., Rincón, G. y Pérez, M. (2005). Initiatives to Face the Logistic Challenges of the Clean Fuel Age: A Software Selection Case, 7thWorld Congress of Chemical Engineering Glasgow, Reino Unido JuPOR. CD: Congress Manuscripts 7o World Congress Chemical Engineering. 1-8.

Álvarez, M., Rincón, G., Pérez, M. y Hernández, S. (2008). Evaluation and Selection of Discrete-Event Simulation Software for the Oil Industry. Latin American Applied Research, 38(4), 305-312.

Antunes, A., Souza, C. y Dutra, L. (2001). Desarrollo de la tecnología en la industria química de Brasil y Venezuela: énfasis en Patentes. Revista Espacios, 22 (22), 1-8.

Durán-García, M. (2007). Propuesta de modelo sistémico para la adopción de la tecnología química. Trabajo de Grado para optar al título de Magíster en Ingeniería de Sistemas. Universidad Simón Bolívar: Caracas.

Durán-García, M., Pérez, M., Rincón, G., Mendoza, L. (2011). Modelo sistémico para la adopción de tecnología por la industria química, DYNA: Ingeniería e Industria, 86 (5), 531-538.

Durán-García, M. (2014). Criterios tecnológicos-Ambientales bajo un enfoque sistémico: transferencia de la tecnología química. Revista Ingeniería, Investigación y Tecnología, 15, 339-350.

Galvis, A. y Vargas, V. (1998). Modelo de selección de tecnología en el tratamiento de agua para consumo humano, Seminario Agua y Sostenibilidad. Conferencia Internacional Colombia, 1-5.

Martínez, A. (1998). Algunas consideraciones sobre la transferencia de tecnología. Revista Tecnología y Sociedad, 1, 114-129.

Mendoza, L., Pérez, M. y Griman, A. (2005). Propuesta del modelo sistémico de calidad (MOSCA) del Software, Revista Computación y Sistemas, 8 (3), 196-221.

Pérez, M., Rojas, T., Mendoza, L. y Griman, A. (2004). Systemic Methodological Framework for is Research, Proceedings of the Tenth Ameritas Conference on Information Systems, 1-15.

Pirela, L. y Paredes, L. (2002). Comportamiento tecnológico de la industria petroquímica venezolana: Caso Pequiven - Unidad de Negocios Olefinas y Plásticos (U.N.O.P.). Revista Espacios, 23 (2), 1-12.

Rincón, G. (2006). Etapas de un Proyecto para la Industria de Procesos. Trabajo de Ascenso a la categoría de Asociado en el área de Procesos Químicos. Departamento de Procesos y Sistemas. Caracas: Universidad Simón Bolívar. 
Rincón, G., Pérez, M., Álvarez, M. y Hernández, S. (2005). A Discrete-Event Simulation and Continuous Software Evaluation on a Systemic Quality Model: an Oil Industry Case. Information and Management, 42 (8), 1051-1061.

Rivera, C. (2002). Análisis termodinámico y planeación operacional de sistemas Generadores de potencia, Trabajo de Grado para optar al título de Maestro en Ciencias en Ingeniería Mecánica. DF México: Instituto Politécnico Nacional.

Turton, R., Bailie, R., Whiting, W., Shaeiwitz, J. y Bhattacharyya, D. (2012). Analysis Synthesis and Design of Chemical Processes. New Jersey: Prentice Hall PTR. 